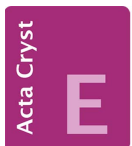

CRYSTALLOGRAPHIC COMMUNICATIONS

ISSN 2056-9890

\section{Crystal structure of cafenstrole}

\author{
Gihaeng Kang, Jineun Kim,* Hyunjin Park and Tae Ho \\ Kim*
}

Department of Chemistry and Research Institute of Natural Sciences, Gyeongsang National University, Jinju 660-701, Republic of Korea. *Correspondence e-mail:

thkim@gnu.ac.kr, jekim@gnu.ac.kr

Received 14 July 2015; accepted 22 July 2015

Edited by P. C. Healy, Griffith University, Australia

The title compound (systematic name: $N, N$-diethyl-3-mesitylsulfonyl-1 $H$-1,2,4-triazole-1-carboxamide), $\mathrm{C}_{16} \mathrm{H}_{22} \mathrm{~N}_{4} \mathrm{O}_{3} \mathrm{~S}$, is a triazole herbicide. The dihedral angle between the planes of the triazole and benzene ring planes is $88.14(10)^{\circ}$. In the crystal, $\mathrm{C}-\mathrm{H} \cdots \mathrm{O}$ hydrogen bonds and weak $\mathrm{C}-\mathrm{H} \cdots \pi$ interactions link adjacent molecules, forming one-dimensional chains along the $a$ axis.

Keywords: crystal structure; cafenstrole; triazole; herbicide,

CCDC reference: 1414616

\section{Related literature}

For information on the herbicidal properties of the title compound, see: Takahashi et al. (2001). For related crystal structure, see: Ohkata et al. (2002).<smiles>CCN(CC)C(=O)n1cnc(S(=O)(=O)c2c(C)cc(C)cc2C)n1</smiles>

\section{Experimental}

\subsection{Crystal data}

$$
\begin{aligned}
& \mathrm{C}_{16} \mathrm{H}_{22} \mathrm{~N}_{4} \mathrm{O}_{3} \mathrm{~S} \\
& M_{r}=350.43 \\
& \text { Monoclinic, } P 2_{1} / c
\end{aligned}
$$

$\beta=95.290(3)^{\circ}$

$V=1759.12(14) \AA^{3}$

$Z=4$

Mo $K \alpha$ radiation

$\mu=0.21 \mathrm{~mm}^{-1}$

$T=173 \mathrm{~K}$

$0.36 \times 0.30 \times 0.02 \mathrm{~mm}$

\subsection{Data collection}

Bruker APEXII CCD diffractometer

Absorption correction: multi-scan (SADABS; Bruker, 2013)

$T_{\min }=0.929, T_{\max }=0.996$

11871 measured reflections 3385 independent reflections 2760 reflections with $I>2 \sigma(I)$ $R_{\text {int }}=0.033$

\subsection{Refinement}

$R\left[F^{2}>2 \sigma\left(F^{2}\right)\right]=0.051$

$w R\left(F^{2}\right)=0.118$

$S=1.12$

3385 reflections
222 parameters

$\mathrm{H}$-atom parameters constrained

$\Delta \rho_{\max }=0.32{\mathrm{e} \AA^{-3}}^{-3}$

$\Delta \rho_{\min }=-0.35 \mathrm{e}^{-3}$
Table 1

Hydrogen-bond geometry $\left(\AA,^{\circ}\right)$.

\begin{tabular}{lllll}
\hline$D-\mathrm{H} \cdots A$ & $D-\mathrm{H}$ & $\mathrm{H} \cdots A$ & $D \cdots A$ & $D-\mathrm{H} \cdots A$ \\
\hline $\mathrm{C} 11-\mathrm{H} 11 \cdots \mathrm{O} 2^{\mathrm{i}}$ & 0.95 & 2.38 & $3.136(3)$ & 136 \\
$\mathrm{C} 7-\mathrm{H} 7 C \cdots C 1^{1 i}$ & 0.98 & 2.80 & $3.561(3)$ & 135 \\
\hline
\end{tabular}

Symmetry codes: (i) $x-1, y, z$; (ii) $-x+1,-y+1,-z$.

Data collection: APEX2 (Bruker, 2013); cell refinement: SAINT (Bruker, 2013); data reduction: $S A I N T$; $\operatorname{program}(\mathrm{s})$ used to solve structure: SHELXS97 (Sheldrick, 2008); program(s) used to refine structure: SHELXL2013 (Sheldrick, 2015); molecular graphics: DIAMOND (Brandenburg, 2010); software used to prepare material for publication: SHELXTL (Sheldrick, 2008).

\section{Acknowledgements}

This research was supported by the Basic Science Research Program through the National Research Foundation of Korea (NRF) funded by the Ministry of Education, Science and Technology (No. 2015R1D1A4A01020317).

Supporting information for this paper is available from the IUCr electronic archives (Reference: HG5453).

\section{References}

Brandenburg, K. (2010). DIAMOND. Crystal Impact GbR, Bonn, Germany. Bruker (2013). APEX2, SAINT and SADABS. Bruker AXS Inc., Madison, Wisconsin, USA.

Ohkata, K., Yano, T., Kojima, S., Hiraga, Y., Yoshii, T. \& Hori, M. (2002). Bull. Chem. Soc. Jpn, 75, 567-574.

Sheldrick, G. M. (2008). Acta Cryst. A64, 112-122.

Sheldrick, G. M. (2015). Acta Cryst. C71, 3-8.

Takahashi, H., Ohki, A., Kanzaki, M., Tanaka, A., Sato, Y., Matthes, B., Böger, P. \& Wakabayashi, K. (2001). J. Biosci. 56, 781-786. 


\section{supporting information}

Acta Cryst. (2015). E71, o614 [https://doi.org/10.1107/S2056989015013869]

\section{Crystal structure of cafenstrole}

\section{Gihaeng Kang, Jineun Kim, Hyunjin Park and Tae Ho Kim}

\section{S1. Comment}

Cafenstrole, or $N, N$-diethyl-3-mesitylsulfonyl-1 $H$-1,2,4-triazole-1-carboxamide, is a triazole herbicide and has been used for rice cultivation which especially inhibits the germination of grass weeds (Takahashi et al., 2001). However, until now its crystal structure has not been reported. In the title compound (Fig. 1), the dihedral angle between the planes of the triazole ring and the phenyl ring planes is $88.14(10)^{\circ}$. All bond lengths and bond angles are normal and comparable to those observed in similar crystal structure (Ohkata et al., 2002).

In the crystal structure (Fig. 2, Table 1), $\mathrm{C} 11-\mathrm{H} 11 \cdots \mathrm{O} 2$ hydrogen bonds and weak intermolecular $\mathrm{C} 7-\mathrm{H} 7 \mathrm{C} \cdots C g 1(C g 1$ is the centroid of the $\mathrm{C} 1-\mathrm{C} 6$ ring) interactions link adjacent molecules, forming one-dimensional chains along to $a$-axis.

\section{S2. Experimental}

The title compound was purchased from the Dr. Ehrenstorfer GmbH Company. Slow evaporation of a solution in $\mathrm{CH}_{3} \mathrm{CN}$ gave single crystals suitable for X-ray analysis.

\section{S3. Refinement}

All H-atoms were positioned geometrically and refined using a riding model with $\mathrm{d}(\mathrm{C}-\mathrm{H})=0.98 \AA, U_{\text {iso }}=1.5 U_{\text {eq }}(\mathrm{C})$ for methyl group, $\mathrm{d}(\mathrm{C}-\mathrm{H})=0.99 \AA, U_{\text {iso }}=1.2 U_{\text {eq }}(\mathrm{C})$ for $\mathrm{Csp} p^{3}-\mathrm{H}, \mathrm{d}(\mathrm{C}-\mathrm{H})=0.95 \AA, U_{\text {iso }}=1.2 U_{\text {eq }}(\mathrm{C})$ for aromatic $\mathrm{C}-\mathrm{H}$.

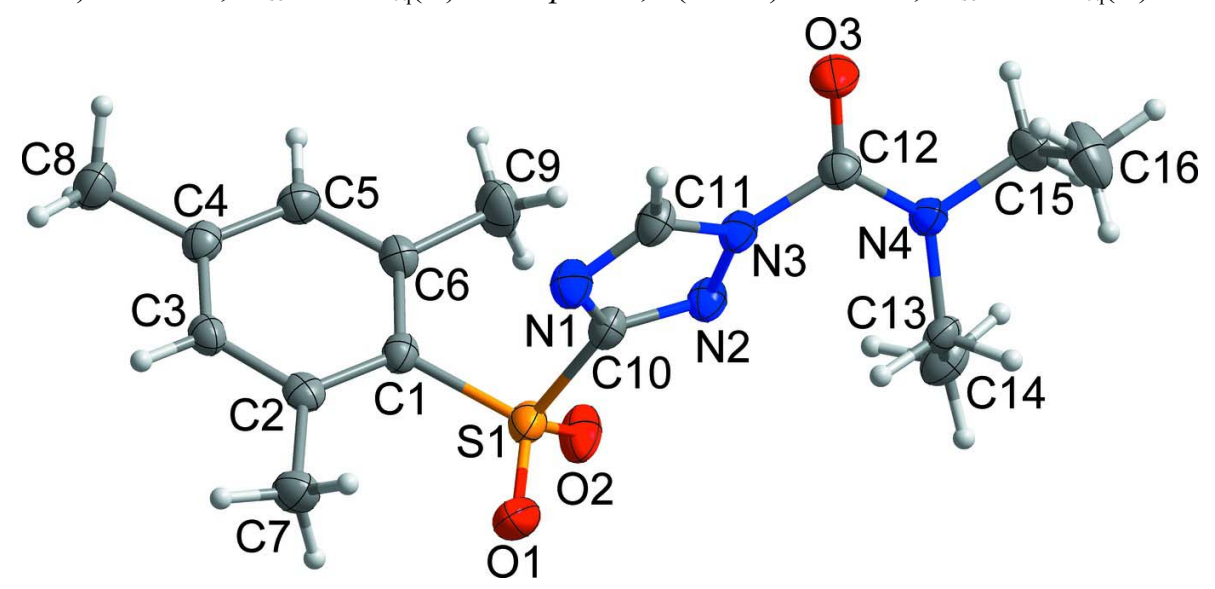

Figure 1

The asymmetric unit of the title compound with the atom numbering scheme. Displacement ellipsoids are drawn at the $50 \%$ probability level. $\mathrm{H}$ atoms are shown as small spheres of arbitrary radius. 


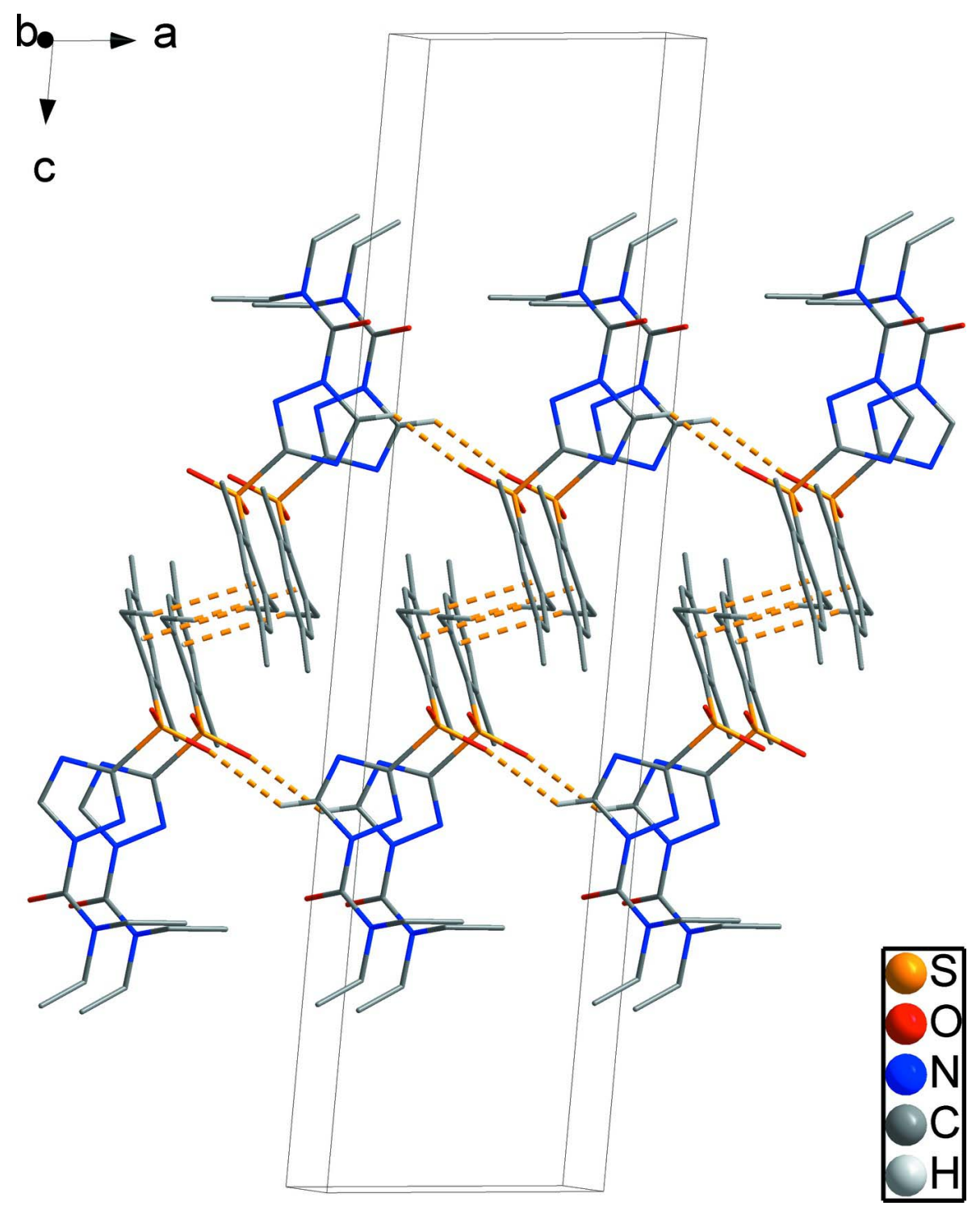

Figure 2

Crystal packing viewed along the $b$ axis. The intermolecular interactions are shown as dashed lines.

N,N-Diethyl-3-mesitylsulfonyl-1H-1,2,4-triazole-1-carboxamide

\section{Crystal data}

$\mathrm{C}_{16} \mathrm{H}_{22} \mathrm{~N}_{4} \mathrm{O}_{3} \mathrm{~S}$

$M_{r}=350.43$

Monoclinic, $P 2{ }_{1} / c$

$a=7.2800(3) \AA$

$b=8.0410(4) \AA$

$c=30.1792(13) \AA$

$\beta=95.290(3)^{\circ}$

$V=1759.12(14) \AA^{3}$

$Z=4$

$F(000)=744$
$D_{\mathrm{x}}=1.323 \mathrm{Mg} \mathrm{m}^{-3}$

Melting point: $390 \mathrm{~K}$

Mo $K \alpha$ radiation, $\lambda=0.71073 \AA$

Cell parameters from 3608 reflections

$\theta=2.6-25.8^{\circ}$

$\mu=0.21 \mathrm{~mm}^{-1}$

$T=173 \mathrm{~K}$

Plate, colourless

$0.36 \times 0.30 \times 0.02 \mathrm{~mm}$ 


\section{Data collection \\ Bruker APEXII CCD \\ diffractometer \\ $\varphi$ and $\omega$ scans \\ (SADABS; Bruker, 2013) \\ $T_{\min }=0.929, T_{\max }=0.996$ \\ 11871 measured reflections \\ Refinement \\ Refinement on $F^{2}$ \\ Least-squares matrix: full \\ $R\left[F^{2}>2 \sigma\left(F^{2}\right)\right]=0.051$ \\ $w R\left(F^{2}\right)=0.118$ \\ $S=1.12$ \\ 3385 reflections \\ 222 parameters \\ 0 restraints}

Absorption correction: multi-scan
3385 independent reflections

2760 reflections with $I>2 \sigma(I)$

$R_{\text {int }}=0.033$

$\theta_{\text {max }}=26.0^{\circ}, \theta_{\min }=1.4^{\circ}$

$h=-8 \rightarrow 7$

$k=-9 \rightarrow 9$

$l=-37 \rightarrow 37$

Hydrogen site location: inferred from neighbouring sites

$\mathrm{H}$-atom parameters constrained

$w=1 /\left[\sigma^{2}\left(F_{\mathrm{o}}^{2}\right)+(0.0373 P)^{2}+1.5378 P\right]$

where $P=\left(F_{\mathrm{o}}{ }^{2}+2 F_{\mathrm{c}}{ }^{2}\right) / 3$

$(\Delta / \sigma)_{\max }<0.001$

$\Delta \rho_{\max }=0.32 \mathrm{e}^{-3}$

$\Delta \rho_{\min }=-0.35$ e $\AA^{-3}$

Special details

Geometry. All e.s.d.'s (except the e.s.d. in the dihedral angle between two 1.s. planes) are estimated using the full covariance matrix. The cell e.s.d.'s are taken into account individually in the estimation of e.s.d.'s in distances, angles and torsion angles; correlations between e.s.d.'s in cell parameters are only used when they are defined by crystal symmetry. An approximate (isotropic) treatment of cell e.s.d.'s is used for estimating e.s.d.'s involving 1.s. planes.

Fractional atomic coordinates and isotropic or equivalent isotropic displacement parameters $\left(\hat{A}^{2}\right)$

\begin{tabular}{lllll}
\hline & $x$ & $y$ & $z$ & $U_{\mathrm{iso}} * / U_{\mathrm{eq}}$ \\
\hline S1 & $0.41370(9)$ & $0.41398(9)$ & $0.10060(2)$ & $0.03098(19)$ \\
O1 & $0.3991(3)$ & $0.2440(2)$ & $0.08694(6)$ & $0.0440(5)$ \\
O2 & $0.5862(2)$ & $0.4698(3)$ & $0.12191(6)$ & $0.0426(5)$ \\
O3 & $-0.0331(3)$ & $0.6067(3)$ & $0.24940(6)$ & $0.0450(5)$ \\
N1 & $0.0629(3)$ & $0.4287(3)$ & $0.12755(6)$ & $0.0321(5)$ \\
N2 & $0.2960(3)$ & $0.4724(3)$ & $0.18146(6)$ & $0.0280(5)$ \\
N3 & $0.1290(3)$ & $0.4783(3)$ & $0.19847(6)$ & $0.0262(5)$ \\
N4 & $0.2305(3)$ & $0.4731(3)$ & $0.27538(6)$ & $0.0265(5)$ \\
C1 & $0.3372(3)$ & $0.5499(3)$ & $0.05661(7)$ & $0.0243(5)$ \\
C2 & $0.2701(3)$ & $0.4890(3)$ & $0.01447(7)$ & $0.0250(5)$ \\
C3 & $0.2198(3)$ & $0.6048(3)$ & $-0.01847(7)$ & $0.0262(5)$ \\
H3 & 0.1760 & 0.5657 & -0.0472 & $0.031^{*}$ \\
C4 & $0.2303(3)$ & $0.7740(3)$ & $-0.01148(8)$ & $0.0275(6)$ \\
C5 & $0.2917(3)$ & $0.8308(3)$ & $0.03064(8)$ & $0.0293(6)$ \\
H5 & 0.2964 & 0.9472 & 0.0360 & $0.035^{*}$ \\
C6 & $0.3468(3)$ & $0.7227(3)$ & $0.06518(7)$ & $0.0272(6)$ \\
C7 & $0.2494(4)$ & $0.3081(3)$ & $0.00170(8)$ & $0.0348(6)$ \\
H7A & 0.1922 & 0.2994 & -0.0289 & $0.052^{*}$ \\
H7B & 0.1713 & 0.2521 & 0.0219 & $0.052^{*}$ \\
H7C & 0.3711 & 0.2552 & 0.0038 & $0.052^{*}$ \\
C8 & $0.1789(4)$ & $0.8928(4)$ & $-0.04883(8)$ & $0.0389(7)$ \\
H8A & 0.2902 & 0.9482 & -0.0574 & $0.058^{*}$ \\
& & & &
\end{tabular}




$\begin{array}{lllll}\text { H8B } & 0.0931 & 0.9763 & -0.0391 & 0.058^{*} \\ \text { H8C } & 0.1199 & 0.8317 & -0.0744 & 0.058^{*} \\ \text { C9 } & 0.4143(4) & 0.7991(4) & 0.10936(8) & 0.0456(8) \\ \text { H9A } & 0.5462 & 0.7758 & 0.1159 & 0.068^{*} \\ \text { H9B } & 0.3459 & 0.7514 & 0.1328 & 0.068^{*} \\ \text { H9C } & 0.3947 & 0.9196 & 0.1081 & 0.068^{*} \\ \text { C10 } & 0.2465(3) & 0.4418(3) & 0.13928(7) & 0.0263(5) \\ \text { C11 } & -0.0062(3) & 0.4550(3) & 0.16559(8) & 0.0302(6) \\ \text { H11 } & -0.1343 & 0.4573 & 0.1694 & 0.036^{*} \\ \text { C12 } & 0.1025(3) & 0.5235(3) & 0.24394(8) & 0.0293(6) \\ \text { C13 } & 0.3683(3) & 0.3424(3) & 0.27089(8) & 0.0253(5) \\ \text { H13A } & 0.3367 & 0.2817 & 0.2427 & 0.030^{*} \\ \text { H13B } & 0.3635 & 0.2621 & 0.2956 & 0.030^{*} \\ \text { C14 } & 0.5623(3) & 0.4096(4) & 0.27125(9) & 0.0364(6) \\ \text { H14A } & 0.5731 & 0.4752 & 0.2443 & 0.055^{*} \\ \text { H14B } & 0.6499 & 0.3169 & 0.2723 & 0.055^{*} \\ \text { H14C } & 0.5896 & 0.4803 & 0.2975 & 0.055^{*} \\ \text { C15 } & 0.2014(4) & 0.5244(4) & 0.32107(8) & 0.0371(7) \\ \text { H15A } & 0.1512 & 0.6389 & 0.3205 & 0.045^{*} \\ \text { H15B } & 0.3214 & 0.5253 & 0.3394 & 0.045^{*} \\ \text { C16 } & 0.0700(4) & 0.4095(5) & 0.34227(9) & 0.0528(9) \\ \text { H16A } & -0.0498 & 0.4096 & 0.3245 & 0.079^{*} \\ \text { H16B } & 0.0542 & 0.4480 & 0.3725 & 0.079^{*} \\ \text { H16C } & 0.1204 & 0.2964 & 0.3435 & 0.079^{*}\end{array}$

Atomic displacement parameters $\left(\AA^{2}\right)$

\begin{tabular}{lllllll}
\hline & $U^{11}$ & $U^{22}$ & $U^{33}$ & $U^{12}$ & $U^{13}$ & $U^{23}$ \\
\hline S1 & $0.0289(4)$ & $0.0379(4)$ & $0.0261(3)$ & $0.0080(3)$ & $0.0024(2)$ & $0.0056(3)$ \\
O1 & $0.0604(13)$ & $0.0328(11)$ & $0.0394(10)$ & $0.0158(10)$ & $0.0082(9)$ & $0.0063(9)$ \\
O2 & $0.0237(10)$ & $0.0704(15)$ & $0.0327(9)$ & $0.0075(10)$ & $-0.0024(7)$ & $0.0098(10)$ \\
O3 & $0.0377(11)$ & $0.0587(14)$ & $0.0388(10)$ & $0.0233(10)$ & $0.0043(8)$ & $0.0019(10)$ \\
N1 & $0.0265(11)$ & $0.0399(14)$ & $0.0290(10)$ & $-0.0012(10)$ & $-0.0020(9)$ & $0.0017(10)$ \\
N2 & $0.0220(11)$ & $0.0345(12)$ & $0.0275(10)$ & $0.0021(9)$ & $0.0020(8)$ & $0.0046(10)$ \\
N3 & $0.0213(11)$ & $0.0306(12)$ & $0.0264(10)$ & $0.0019(9)$ & $0.0000(8)$ & $0.0035(9)$ \\
N4 & $0.0290(11)$ & $0.0244(11)$ & $0.0251(10)$ & $0.0033(9)$ & $-0.0031(8)$ & $-0.0011(9)$ \\
C1 & $0.0214(12)$ & $0.0284(13)$ & $0.0230(11)$ & $0.0013(11)$ & $0.0018(9)$ & $0.0029(11)$ \\
C2 & $0.0173(12)$ & $0.0319(14)$ & $0.0261(11)$ & $-0.0003(10)$ & $0.0037(9)$ & $-0.0022(11)$ \\
C3 & $0.0241(12)$ & $0.0326(14)$ & $0.0215(11)$ & $0.0007(11)$ & $0.0003(9)$ & $-0.0030(11)$ \\
C4 & $0.0233(13)$ & $0.0316(14)$ & $0.0276(12)$ & $0.0018(11)$ & $0.0026(10)$ & $0.0034(11)$ \\
C5 & $0.0321(14)$ & $0.0252(13)$ & $0.0309(12)$ & $-0.0004(11)$ & $0.0043(11)$ & $-0.0005(11)$ \\
C6 & $0.0272(13)$ & $0.0318(14)$ & $0.0226(11)$ & $-0.0017(11)$ & $0.0016(10)$ & $-0.0004(11)$ \\
C7 & $0.0390(16)$ & $0.0295(15)$ & $0.0353(13)$ & $0.0013(13)$ & $0.0002(11)$ & $-0.0050(12)$ \\
C8 & $0.0479(17)$ & $0.0350(16)$ & $0.0328(13)$ & $0.0050(14)$ & $-0.0023(12)$ & $0.0057(13)$ \\
C9 & $0.066(2)$ & $0.0384(17)$ & $0.0306(14)$ & $-0.0064(16)$ & $-0.0051(13)$ & $-0.0052(13)$ \\
C10 & $0.0267(13)$ & $0.0270(13)$ & $0.0246(11)$ & $0.0018(11)$ & $-0.0011(10)$ & $0.0046(11)$ \\
C11 & $0.0256(13)$ & $0.0338(15)$ & $0.0299(12)$ & $0.0007(11)$ & $-0.0034(10)$ & $0.0067(12)$ \\
C12 & $0.0253(14)$ & $0.0319(14)$ & $0.0306(13)$ & $0.0029(12)$ & $0.0022(10)$ & $0.0014(11)$
\end{tabular}




\begin{tabular}{lllllll}
$\mathrm{C} 13$ & $0.0276(13)$ & $0.0210(12)$ & $0.0265(11)$ & $0.0040(10)$ & $-0.0014(10)$ & $0.0047(10)$ \\
$\mathrm{C} 14$ & $0.0278(14)$ & $0.0360(15)$ & $0.0441(15)$ & $-0.0003(12)$ & $-0.0026(11)$ & $0.0088(13)$ \\
$\mathrm{C} 15$ & $0.0461(17)$ & $0.0354(15)$ & $0.0281(13)$ & $0.0066(13)$ & $-0.0057(12)$ & $-0.0099(12)$ \\
$\mathrm{C} 16$ & $0.0481(18)$ & $0.083(3)$ & $0.0285(13)$ & $-0.0110(18)$ & $0.0087(13)$ & $-0.0122(16)$ \\
\hline
\end{tabular}

Geometric parameters $\left(\AA,{ }^{o}\right)$

\begin{tabular}{|c|c|c|c|}
\hline $\mathrm{S} 1-\mathrm{O} 1$ & $1.429(2)$ & $\mathrm{C} 6-\mathrm{C} 9$ & $1.509(3)$ \\
\hline $\mathrm{S} 1-\mathrm{O} 2$ & $1.4293(19)$ & C7-H7A & 0.9800 \\
\hline $\mathrm{S} 1-\mathrm{C} 1$ & $1.769(2)$ & C7-H7B & 0.9800 \\
\hline $\mathrm{S} 1-\mathrm{C} 10$ & $1.777(2)$ & $\mathrm{C} 7-\mathrm{H} 7 \mathrm{C}$ & 0.9800 \\
\hline $\mathrm{O} 3-\mathrm{C} 12$ & $1.216(3)$ & $\mathrm{C} 8-\mathrm{H} 8 \mathrm{~A}$ & 0.9800 \\
\hline $\mathrm{N} 1-\mathrm{C} 11$ & $1.313(3)$ & $\mathrm{C} 8-\mathrm{H} 8 \mathrm{~B}$ & 0.9800 \\
\hline $\mathrm{N} 1-\mathrm{C} 10$ & $1.355(3)$ & $\mathrm{C} 8-\mathrm{H} 8 \mathrm{C}$ & 0.9800 \\
\hline $\mathrm{N} 2-\mathrm{C} 10$ & $1.314(3)$ & C9-H9A & 0.9800 \\
\hline $\mathrm{N} 2-\mathrm{N} 3$ & $1.364(3)$ & C9-H9B & 0.9800 \\
\hline N3-C11 & $1.345(3)$ & C9- $\mathrm{H} 9 \mathrm{C}$ & 0.9800 \\
\hline $\mathrm{N} 3-\mathrm{C} 12$ & $1.450(3)$ & C11-H11 & 0.9500 \\
\hline $\mathrm{N} 4-\mathrm{C} 12$ & $1.331(3)$ & $\mathrm{C} 13-\mathrm{C} 14$ & $1.511(3)$ \\
\hline $\mathrm{N} 4-\mathrm{C} 13$ & $1.468(3)$ & $\mathrm{C} 13-\mathrm{H} 13 \mathrm{~A}$ & 0.9900 \\
\hline $\mathrm{N} 4-\mathrm{C} 15$ & $1.473(3)$ & C13-H13B & 0.9900 \\
\hline $\mathrm{C} 1-\mathrm{C} 2$ & $1.408(3)$ & $\mathrm{C} 14-\mathrm{H} 14 \mathrm{~A}$ & 0.9800 \\
\hline $\mathrm{C} 1-\mathrm{C} 6$ & $1.414(4)$ & $\mathrm{C} 14-\mathrm{H} 14 \mathrm{~B}$ & 0.9800 \\
\hline $\mathrm{C} 2-\mathrm{C} 3$ & $1.387(3)$ & $\mathrm{C} 14-\mathrm{H} 14 \mathrm{C}$ & 0.9800 \\
\hline $\mathrm{C} 2-\mathrm{C} 7$ & $1.509(3)$ & $\mathrm{C} 15-\mathrm{C} 16$ & $1.513(4)$ \\
\hline $\mathrm{C} 3-\mathrm{C} 4$ & $1.378(4)$ & C15-H15A & 0.9900 \\
\hline $\mathrm{C} 3-\mathrm{H} 3$ & 0.9500 & C15-H15B & 0.9900 \\
\hline $\mathrm{C} 4-\mathrm{C} 5$ & $1.385(3)$ & $\mathrm{C} 16-\mathrm{H} 16 \mathrm{~A}$ & 0.9800 \\
\hline $\mathrm{C} 4-\mathrm{C} 8$ & $1.499(3)$ & $\mathrm{C} 16-\mathrm{H} 16 \mathrm{~B}$ & 0.9800 \\
\hline $\mathrm{C} 5-\mathrm{C} 6$ & $1.387(3)$ & $\mathrm{C} 16-\mathrm{H} 16 \mathrm{C}$ & 0.9800 \\
\hline $\mathrm{C} 5-\mathrm{H} 5$ & 0.9500 & & \\
\hline $\mathrm{O} 1-\mathrm{S} 1-\mathrm{O} 2$ & $118.01(13)$ & $\mathrm{H} 8 \mathrm{~A}-\mathrm{C} 8-\mathrm{H} 8 \mathrm{C}$ & 109.5 \\
\hline $\mathrm{O} 1-\mathrm{S} 1-\mathrm{C} 1$ & $111.37(11)$ & $\mathrm{H} 8 \mathrm{~B}-\mathrm{C} 8-\mathrm{H} 8 \mathrm{C}$ & 109.5 \\
\hline $\mathrm{O} 2-\mathrm{S} 1-\mathrm{C} 1$ & $110.25(12)$ & $\mathrm{C} 6-\mathrm{C} 9-\mathrm{H} 9 \mathrm{~A}$ & 109.5 \\
\hline $\mathrm{O} 1-\mathrm{S} 1-\mathrm{C} 10$ & $105.83(12)$ & $\mathrm{C} 6-\mathrm{C} 9-\mathrm{H} 9 \mathrm{~B}$ & 109.5 \\
\hline $\mathrm{O} 2-\mathrm{S} 1-\mathrm{C} 10$ & $106.94(11)$ & $\mathrm{H} 9 \mathrm{~A}-\mathrm{C} 9-\mathrm{H} 9 \mathrm{~B}$ & 109.5 \\
\hline $\mathrm{C} 1-\mathrm{S} 1-\mathrm{C} 10$ & $103.17(11)$ & $\mathrm{C} 6-\mathrm{C} 9-\mathrm{H} 9 \mathrm{C}$ & 109.5 \\
\hline $\mathrm{C} 11-\mathrm{N} 1-\mathrm{C} 10$ & $101.98(19)$ & $\mathrm{H} 9 \mathrm{~A}-\mathrm{C} 9-\mathrm{H} 9 \mathrm{C}$ & 109.5 \\
\hline $\mathrm{C} 10-\mathrm{N} 2-\mathrm{N} 3$ & $101.33(18)$ & $\mathrm{H} 9 \mathrm{~B}-\mathrm{C} 9-\mathrm{H} 9 \mathrm{C}$ & 109.5 \\
\hline $\mathrm{C} 11-\mathrm{N} 3-\mathrm{N} 2$ & 109.55 (19) & $\mathrm{N} 2-\mathrm{C} 10-\mathrm{N} 1$ & $116.4(2)$ \\
\hline $\mathrm{C} 11-\mathrm{N} 3-\mathrm{C} 12$ & $125.5(2)$ & $\mathrm{N} 2-\mathrm{C} 10-\mathrm{S} 1$ & $121.10(18)$ \\
\hline $\mathrm{N} 2-\mathrm{N} 3-\mathrm{C} 12$ & 124.48 (19) & $\mathrm{N} 1-\mathrm{C} 10-\mathrm{S} 1$ & $122.51(17)$ \\
\hline $\mathrm{C} 12-\mathrm{N} 4-\mathrm{C} 13$ & $126.3(2)$ & $\mathrm{N} 1-\mathrm{C} 11-\mathrm{N} 3$ & $110.7(2)$ \\
\hline $\mathrm{C} 12-\mathrm{N} 4-\mathrm{C} 15$ & $115.5(2)$ & $\mathrm{N} 1-\mathrm{C} 11-\mathrm{H} 11$ & 124.6 \\
\hline $\mathrm{C} 13-\mathrm{N} 4-\mathrm{C} 15$ & $116.46(19)$ & $\mathrm{N} 3-\mathrm{C} 11-\mathrm{H} 11$ & 124.6 \\
\hline $\mathrm{C} 2-\mathrm{C} 1-\mathrm{C} 6$ & $120.9(2)$ & $\mathrm{O} 3-\mathrm{C} 12-\mathrm{N} 4$ & $126.5(2)$ \\
\hline $\mathrm{C} 2-\mathrm{C} 1-\mathrm{S} 1$ & $121.47(19)$ & $\mathrm{O} 3-\mathrm{C} 12-\mathrm{N} 3$ & $116.5(2)$ \\
\hline
\end{tabular}




\begin{tabular}{|c|c|c|c|}
\hline $\mathrm{C} 6-\mathrm{C} 1-\mathrm{S} 1$ & $117.58(17)$ & $\mathrm{N} 4-\mathrm{C} 12-\mathrm{N} 3$ & $116.9(2)$ \\
\hline $\mathrm{C} 3-\mathrm{C} 2-\mathrm{C} 1$ & $117.4(2)$ & $\mathrm{N} 4-\mathrm{C} 13-\mathrm{C} 14$ & $112.9(2)$ \\
\hline $\mathrm{C} 3-\mathrm{C} 2-\mathrm{C} 7$ & $116.8(2)$ & $\mathrm{N} 4-\mathrm{C} 13-\mathrm{H} 13 \mathrm{~A}$ & 109.0 \\
\hline $\mathrm{C} 1-\mathrm{C} 2-\mathrm{C} 7$ & $125.8(2)$ & $\mathrm{C} 14-\mathrm{C} 13-\mathrm{H} 13 \mathrm{~A}$ & 109.0 \\
\hline $\mathrm{C} 4-\mathrm{C} 3-\mathrm{C} 2$ & $123.1(2)$ & N4-C13-H13B & 109.0 \\
\hline $\mathrm{C} 4-\mathrm{C} 3-\mathrm{H} 3$ & 118.4 & $\mathrm{C} 14-\mathrm{C} 13-\mathrm{H} 13 \mathrm{~B}$ & 109.0 \\
\hline $\mathrm{C} 2-\mathrm{C} 3-\mathrm{H} 3$ & 118.4 & $\mathrm{H} 13 \mathrm{~A}-\mathrm{C} 13-\mathrm{H} 13 \mathrm{~B}$ & 107.8 \\
\hline $\mathrm{C} 3-\mathrm{C} 4-\mathrm{C} 5$ & $118.3(2)$ & $\mathrm{C} 13-\mathrm{C} 14-\mathrm{H} 14 \mathrm{~A}$ & 109.5 \\
\hline $\mathrm{C} 3-\mathrm{C} 4-\mathrm{C} 8$ & $120.5(2)$ & $\mathrm{C} 13-\mathrm{C} 14-\mathrm{H} 14 \mathrm{~B}$ & 109.5 \\
\hline $\mathrm{C} 5-\mathrm{C} 4-\mathrm{C} 8$ & $121.1(2)$ & $\mathrm{H} 14 \mathrm{~A}-\mathrm{C} 14-\mathrm{H} 14 \mathrm{~B}$ & 109.5 \\
\hline $\mathrm{C} 4-\mathrm{C} 5-\mathrm{C} 6$ & $122.0(2)$ & $\mathrm{C} 13-\mathrm{C} 14-\mathrm{H} 14 \mathrm{C}$ & 109.5 \\
\hline $\mathrm{C} 4-\mathrm{C} 5-\mathrm{H} 5$ & 119.0 & $\mathrm{H} 14 \mathrm{~A}-\mathrm{C} 14-\mathrm{H} 14 \mathrm{C}$ & 109.5 \\
\hline $\mathrm{C} 6-\mathrm{C} 5-\mathrm{H} 5$ & 119.0 & $\mathrm{H} 14 \mathrm{~B}-\mathrm{C} 14-\mathrm{H} 14 \mathrm{C}$ & 109.5 \\
\hline $\mathrm{C} 5-\mathrm{C} 6-\mathrm{C} 1$ & $118.2(2)$ & $\mathrm{N} 4-\mathrm{C} 15-\mathrm{C} 16$ & $112.1(2)$ \\
\hline $\mathrm{C} 5-\mathrm{C} 6-\mathrm{C} 9$ & $117.2(2)$ & $\mathrm{N} 4-\mathrm{C} 15-\mathrm{H} 15 \mathrm{~A}$ & 109.2 \\
\hline $\mathrm{C} 1-\mathrm{C} 6-\mathrm{C} 9$ & $124.6(2)$ & $\mathrm{C} 16-\mathrm{C} 15-\mathrm{H} 15 \mathrm{~A}$ & 109.2 \\
\hline $\mathrm{C} 2-\mathrm{C} 7-\mathrm{H} 7 \mathrm{~A}$ & 109.5 & N4-C15-H15B & 109.2 \\
\hline $\mathrm{C} 2-\mathrm{C} 7-\mathrm{H} 7 \mathrm{~B}$ & 109.5 & $\mathrm{C} 16-\mathrm{C} 15-\mathrm{H} 15 \mathrm{~B}$ & 109.2 \\
\hline $\mathrm{H} 7 \mathrm{~A}-\mathrm{C} 7-\mathrm{H} 7 \mathrm{~B}$ & 109.5 & $\mathrm{H} 15 \mathrm{~A}-\mathrm{C} 15-\mathrm{H} 15 \mathrm{~B}$ & 107.9 \\
\hline $\mathrm{C} 2-\mathrm{C} 7-\mathrm{H} 7 \mathrm{C}$ & 109.5 & $\mathrm{C} 15-\mathrm{C} 16-\mathrm{H} 16 \mathrm{~A}$ & 109.5 \\
\hline $\mathrm{H} 7 \mathrm{~A}-\mathrm{C} 7-\mathrm{H} 7 \mathrm{C}$ & 109.5 & $\mathrm{C} 15-\mathrm{C} 16-\mathrm{H} 16 \mathrm{~B}$ & 109.5 \\
\hline $\mathrm{H} 7 \mathrm{~B}-\mathrm{C} 7-\mathrm{H} 7 \mathrm{C}$ & 109.5 & $\mathrm{H} 16 \mathrm{~A}-\mathrm{C} 16-\mathrm{H} 16 \mathrm{~B}$ & 109.5 \\
\hline $\mathrm{C} 4-\mathrm{C} 8-\mathrm{H} 8 \mathrm{~A}$ & 109.5 & $\mathrm{C} 15-\mathrm{C} 16-\mathrm{H} 16 \mathrm{C}$ & 109.5 \\
\hline $\mathrm{C} 4-\mathrm{C} 8-\mathrm{H} 8 \mathrm{~B}$ & 109.5 & $\mathrm{H} 16 \mathrm{~A}-\mathrm{C} 16-\mathrm{H} 16 \mathrm{C}$ & 109.5 \\
\hline $\mathrm{H} 8 \mathrm{~A}-\mathrm{C} 8-\mathrm{H} 8 \mathrm{~B}$ & 109.5 & $\mathrm{H} 16 \mathrm{~B}-\mathrm{C} 16-\mathrm{H} 16 \mathrm{C}$ & 109.5 \\
\hline $\mathrm{C} 4-\mathrm{C} 8-\mathrm{H} 8 \mathrm{C}$ & 109.5 & & \\
\hline $\mathrm{C} 10-\mathrm{N} 2-\mathrm{N} 3-\mathrm{C} 11$ & $-1.4(3)$ & $\mathrm{N} 3-\mathrm{N} 2-\mathrm{C} 10-\mathrm{S} 1$ & $-177.87(17)$ \\
\hline $\mathrm{C} 10-\mathrm{N} 2-\mathrm{N} 3-\mathrm{C} 12$ & $-174.3(2)$ & $\mathrm{C} 11-\mathrm{N} 1-\mathrm{C} 10-\mathrm{N} 2$ & $0.7(3)$ \\
\hline $\mathrm{O} 1-\mathrm{S} 1-\mathrm{C} 1-\mathrm{C} 2$ & $0.7(2)$ & $\mathrm{C} 11-\mathrm{N} 1-\mathrm{C} 10-\mathrm{S} 1$ & $178.95(19)$ \\
\hline $\mathrm{O} 2-\mathrm{S} 1-\mathrm{C} 1-\mathrm{C} 2$ & $133.7(2)$ & $\mathrm{O} 1-\mathrm{S} 1-\mathrm{C} 10-\mathrm{N} 2$ & $113.2(2)$ \\
\hline $\mathrm{C} 10-\mathrm{S} 1-\mathrm{C} 1-\mathrm{C} 2$ & $-112.4(2)$ & $\mathrm{O} 2-\mathrm{S} 1-\mathrm{C} 10-\mathrm{N} 2$ & $-13.4(2)$ \\
\hline $\mathrm{O} 1-\mathrm{S} 1-\mathrm{C} 1-\mathrm{C} 6$ & $-178.97(18)$ & $\mathrm{C} 1-\mathrm{S} 1-\mathrm{C} 10-\mathrm{N} 2$ & $-129.7(2)$ \\
\hline $\mathrm{O} 2-\mathrm{S} 1-\mathrm{C} 1-\mathrm{C} 6$ & $-46.0(2)$ & $\mathrm{O} 1-\mathrm{S} 1-\mathrm{C} 10-\mathrm{N} 1$ & $-65.0(2)$ \\
\hline $\mathrm{C} 10-\mathrm{S} 1-\mathrm{C} 1-\mathrm{C} 6$ & $67.9(2)$ & $\mathrm{O} 2-\mathrm{S} 1-\mathrm{C} 10-\mathrm{N} 1$ & $168.3(2)$ \\
\hline $\mathrm{C} 6-\mathrm{C} 1-\mathrm{C} 2-\mathrm{C} 3$ & $2.1(3)$ & $\mathrm{C} 1-\mathrm{S} 1-\mathrm{C} 10-\mathrm{N} 1$ & $52.1(2)$ \\
\hline $\mathrm{S} 1-\mathrm{C} 1-\mathrm{C} 2-\mathrm{C} 3$ & $-177.58(17)$ & $\mathrm{C} 10-\mathrm{N} 1-\mathrm{C} 11-\mathrm{N} 3$ & $-1.5(3)$ \\
\hline $\mathrm{C} 6-\mathrm{C} 1-\mathrm{C} 2-\mathrm{C} 7$ & $-178.5(2)$ & $\mathrm{N} 2-\mathrm{N} 3-\mathrm{C} 11-\mathrm{N} 1$ & $2.0(3)$ \\
\hline $\mathrm{S} 1-\mathrm{C} 1-\mathrm{C} 2-\mathrm{C} 7$ & $1.8(3)$ & $\mathrm{C} 12-\mathrm{N} 3-\mathrm{C} 11-\mathrm{N} 1$ & $174.8(2)$ \\
\hline $\mathrm{C} 1-\mathrm{C} 2-\mathrm{C} 3-\mathrm{C} 4$ & $-1.0(4)$ & $\mathrm{C} 13-\mathrm{N} 4-\mathrm{C} 12-\mathrm{O} 3$ & $164.5(3)$ \\
\hline $\mathrm{C} 7-\mathrm{C} 2-\mathrm{C} 3-\mathrm{C} 4$ & $179.6(2)$ & $\mathrm{C} 15-\mathrm{N} 4-\mathrm{C} 12-\mathrm{O} 3$ & $0.2(4)$ \\
\hline $\mathrm{C} 2-\mathrm{C} 3-\mathrm{C} 4-\mathrm{C} 5$ & $-0.9(4)$ & $\mathrm{C} 13-\mathrm{N} 4-\mathrm{C} 12-\mathrm{N} 3$ & $-17.0(4)$ \\
\hline $\mathrm{C} 2-\mathrm{C} 3-\mathrm{C} 4-\mathrm{C} 8$ & $178.2(2)$ & $\mathrm{C} 15-\mathrm{N} 4-\mathrm{C} 12-\mathrm{N} 3$ & $178.7(2)$ \\
\hline $\mathrm{C} 3-\mathrm{C} 4-\mathrm{C} 5-\mathrm{C} 6$ & $1.7(4)$ & $\mathrm{C} 11-\mathrm{N} 3-\mathrm{C} 12-\mathrm{O} 3$ & $-29.9(4)$ \\
\hline $\mathrm{C} 8-\mathrm{C} 4-\mathrm{C} 5-\mathrm{C} 6$ & $-177.4(2)$ & $\mathrm{N} 2-\mathrm{N} 3-\mathrm{C} 12-\mathrm{O} 3$ & $141.9(3)$ \\
\hline $\mathrm{C} 4-\mathrm{C} 5-\mathrm{C} 6-\mathrm{C} 1$ & $-0.6(4)$ & $\mathrm{C} 11-\mathrm{N} 3-\mathrm{C} 12-\mathrm{N} 4$ & $151.5(2)$ \\
\hline $\mathrm{C} 4-\mathrm{C} 5-\mathrm{C} 6-\mathrm{C} 9$ & $178.9(2)$ & $\mathrm{N} 2-\mathrm{N} 3-\mathrm{C} 12-\mathrm{N} 4$ & $-36.8(3)$ \\
\hline $\mathrm{C} 2-\mathrm{C} 1-\mathrm{C} 6-\mathrm{C} 5$ & $-1.3(4)$ & $\mathrm{C} 12-\mathrm{N} 4-\mathrm{C} 13-\mathrm{C} 14$ & $110.4(3)$ \\
\hline
\end{tabular}




$\begin{array}{llll}\mathrm{S} 1-\mathrm{C} 1-\mathrm{C} 6-\mathrm{C} 5 & 178.35(18) & \mathrm{C} 15-\mathrm{N} 4-\mathrm{C} 13-\mathrm{C} 14 & -85.5(3) \\ \mathrm{C} 2-\mathrm{C} 1-\mathrm{C} 6-\mathrm{C} 9 & 179.2(2) & \mathrm{C} 12-\mathrm{N} 4-\mathrm{C} 15-\mathrm{C} 16 & 82.9(3) \\ \mathrm{S} 1-\mathrm{C} 1-\mathrm{C} 6-\mathrm{C} 9 & -1.2(3) & \mathrm{C} 13-\mathrm{N} 4-\mathrm{C} 15-\mathrm{C} 16 & -83.0(3) \\ \mathrm{N} 3-\mathrm{N} 2-\mathrm{C} 10-\mathrm{N} 1 & 0.4(3) & & \end{array}$

Hydrogen-bond geometry $\left(\AA,{ }^{\circ}\right)$

\begin{tabular}{lllll}
\hline$D-\mathrm{H} \cdots A$ & $D-\mathrm{H}$ & $\mathrm{H} \cdots A$ & $D \cdots A$ & $D-\mathrm{H} \cdots A$ \\
\hline $\mathrm{C} 11-\mathrm{H} 11 \cdots \mathrm{O} 2^{\mathrm{i}}$ & 0.95 & 2.38 & $3.136(3)$ & 136 \\
$\mathrm{C} 7-\mathrm{H} 7 C \cdots C g 1^{\mathrm{ii}}$ & 0.98 & 2.80 & $3.561(3)$ & 135 \\
\hline
\end{tabular}

Symmetry codes: (i) $x-1, y, z$; (ii) $-x+1,-y+1,-z$. 